\title{
Time dependence of breakdown in a global fiber-bundle model with continuous damage
}

\author{
L. Moral ${ }^{1}$, Y. Moreno ${ }^{2}$, J.B.Gómez ${ }^{1}$, A. F. Pacheco ${ }^{1}$ \\ ${ }^{1}$ Facultad de Ciencias, Universidad de Zaragoza, 50009 Zaragoza, Spain. \\ 2 The Abdus Salam International Centre for Theoretical Physics, \\ Condensed Matter Group, P.O. Box 586, Trieste, I-34014, Italy.
}

(October 25, 2018)

\begin{abstract}
A time-dependent global fiber-bundle model of fracture with continuous damage is formulated in terms of a set of coupled non-linear differential equations. A first integral of this set is analytically obtained. The time evolution of the system is studied by applying a discrete probabilistic method. Several results are discussed emphasizing their differences with the standard time-dependent model. The results obtained show that with this simple model a variety of experimental observations can be qualitatively reproduced.

PACS number(s): 46.50.+a, 62.20.Fe, 62.20.Mk
\end{abstract}

Fracture in disordered media has attracted much scientific and industrial interest for many years [1]7]; it is, however, a complex problem for which a definite physical and theoretical treatment is still lacking. An important class of models of material failure is the fiberbundle models (FBM) which have been extensively studied during the past decades [7 12]. These models consist of a set of parallel fibers having statistically distributed strength. The sample is loaded parallel to the fiber direction, and a fiber fails if the load acting on it exceeds a threshold value. When a fiber fails, its load is transferred to other surviving fibers in the bundle, according to a specific transfer rule. Among the possible options of load transfer, one simplification that makes the problem analytically tractable is the assumption of equal load sharing (ELS), or global load transfer, which means that after each fiber breaks, its stress is equally distributed among the intact fibers. Thus, the ELS option constitutes a sort of mean field approximation to other more realistic rules of stress transfer where a stress enhancement occurs in the neighborhood of failed elements. So far, the failure rule applied in standard FBM is discontinuous and irreversible, i.e., when the local load exceeds the failure threshold of a fiber, the fiber is removed from the calculation and is never restored. Recently, a novel continuous damage law was introduced in these models 113,14. Thus, when the strength threshold of a fiber is exceeded, it yields, and the elastic modulus of the fiber is reduced by a factor $a(0<a<1)$. Multiple yields of a given fiber are allowed. It is argued that this description of damage in terms of a continuous parameter corresponds to the consideration of the system at a length scale larger than the typical crack size; i.e., if the smallest elements of the model are the fibers, the continuous damage is due to cracking inside the fibers. This generalization of the standard FBM is suitable to describe a variety of elasto-plastic constitutive behaviors 15 177.

FBM come in two settings, static and time-dependent or dynamic. The static version of FBM simulates the fail- ure of materials by quasiestatic loading, i.e, by a steady increase in the load over the system up to its macroscopic failure. The stress on each fiber is the independent variable and the strength of each element is the distributed random variable. On the other hand, the dynamic FBM simulates failure by creep rupture, static fatigue, or delayed rupture, i.e., a (usually) constant load is imposed on the system and the elements break because of fatigue after a period of time. The time elapsed until the system collapses is the lifetime of the bundle. Time acts as an independent variable, and the initial lifetime of each element, for a prescribed initial stress, is the independent identically distributed random quantity.

The concept of continuous damage in FBM has only been applied to the static setting. However, timedependent mechanisms also play a key role in the process of fracture. Phenomena such as fatigue and stress corrosion are of utmost importance for real applications. These time-dependent effects have not been included before in continuous damage descriptions. So, the precise purpose of this paper is to formulate for the first time, the time-dependent FBM with continuous damage and compute the differences appearing with respect to the standard dynamic FBM.

In these models the most widely used breaking rate function is the power law 12, in which elements break at a rate proportional to a power of their stress, $\sigma^{\rho}$, where the exponent $\rho$ is an integer called the stress corrosion exponent. This type of breaking rate will be assumed here.

Our analysis will be restricted to the global transfer modality, and we will assume that the size of the bundle, $N$, is very large. This enables us to formulate the evolution of the system in terms of continuous differential equations. This type of equations, similar to those appearing in radioactivity, was first used by Coleman [8], and later in [11. At this point, it is worth recalling that for the standard model, the lifetime $T$ of the bundle can be analytically obtained. In this case, the differential 
equation governing the time evolution of the system reads

$$
\frac{d N_{0}}{d t}=-N_{0} \cdot f^{\rho},
$$

where $f=\sigma=N / N_{0}$ is the strain of the bundle assuming that the elastic modulus of the fibers is $Y=1$ and $\sigma$ is the individual stress acting on one fiber. The solution of Eq. (11) should fulfill the condition $N_{0}(t=0)=N$. The integration of (1) is straightforward and the lifetime of the bundle is given by $T=\frac{1}{\rho}$.

Now, suppose an ELS bundle formed by $N$ fibers which breaks because of stress corrosion under the action of an external constant load $F=N \cdot \sigma_{0}$, with $\sigma_{0}=1$. The breaking rate of the fibers, $\Gamma$, is assumed to be of the power-law type, $\Gamma=\sigma^{\rho}$. As before, $f$ will denote the strain of the bundle and $Y=1$ will represent the initial stiffness of the individual fibers. The original dynamic FBM is generalized by allowing that one fiber can fail more than once, and thus we define the integer $n$ as the maximum value of failures allowed per fiber. Besides, the parameter $a(<1)$ will represent the factor of reduction in the stiffness of the fibers when they fail. As up to $n$ partial yielding events are permitted per fiber, at any time the population of fibers will be sorted in $n+2$ lists. Thus $N=N_{0}+N_{1}+\ldots+N_{n}+N^{\prime}$, where $N_{j}$ $(j=0, \ldots, n)$ denotes the number of elements that have failed $j$ times. $N^{\prime}$ denotes the number of elements that have failed $n+1$ times and therefore are inactive (i.e., they do not support load anymore). At $t=0$, the $N$ elements of the bundle form the list $0, N_{0}=N$, and at $t=T, N^{\prime}=N$. The specification, at a given time $t$, of the value of $N_{j}$, for $j=0,1, \ldots, n$, provides the state of the system. In our continuous formulation the $N_{j}$ will be real positive numbers lower than $N$.

As the external load $F=N$ is supported by the present active fibers, we have

$$
N=f \cdot\left(N_{0}+a N_{1}+a^{2} N_{2}+\ldots+a^{n} N_{n}\right),
$$

and hence $f=N /\left(N_{0}+a N_{1}+a^{2} N_{2}+\ldots+a^{n} N_{n}\right)$.

The time evolution equations are:

$$
\begin{array}{r}
\frac{d N_{0}}{d t}=f^{\rho}\left(-N_{0}\right), \\
\frac{d N_{1}}{d t}=f^{\rho}\left(N_{0}-k N_{1}\right), \\
\frac{d N_{2}}{d t}=f^{\rho} k\left(N_{1}-k N_{2}\right), \\
\vdots \\
\frac{d N_{n}}{d t}=f^{\rho} k^{n-1}\left(N_{n-1}-k N_{n}\right),
\end{array}
$$

where the ubiquitous constant factor $k$ represents $k=a^{\rho}$. This is a system of coupled, first-order, non-linear differential equations. Its solution must fulfill the initial condition

$$
\begin{array}{r}
N_{0}(t=0)=N \\
N_{j}(t=0)=0, \quad j \neq 0 .
\end{array}
$$

On the right-hand side of Eq. (3), the positive terms represent the sources and the negative ones represent the sinks of the various lists. Eq. (3) does not have an analytic solution. However a first integral can be given expressing $N_{j}(j \neq 0)$ in terms of $N_{0}$. The source of nonlinearity in Eq. (3) is the factor $f^{\rho}$ on the right-hand side. This factor can be eliminated by reformulating the system of equations in such a way that $N_{0}$ is the new independent variable and $N_{j}(j \neq 0)$ the dependent variables. Denoting by a prime the derivative with respect to $N_{0}$, one easily obtains

$$
\begin{array}{r}
N_{1}^{\prime}=\left(k N_{1}-N_{0}\right) / N_{0}, \\
N_{2}^{\prime}=k\left(k N_{2}-N_{1}\right) / N_{0}, \\
\vdots \\
N_{n}^{\prime}=k^{n-1}\left(k N_{n}-N_{n-1}\right) / N_{0} .
\end{array}
$$

This coupled set of first-order linear differential equations must fulfill

$$
N_{j}\left(N_{0}=N\right)=0, \quad j \neq 0
$$

as initial conditions.

The solutions of Eq. (5) fulfilling Eq. (6) for an arbitrary index $l, l=1,2, \ldots, n$, are

$$
N_{l}=\sum_{j=0}^{l} a_{j}^{(l)} N_{0}^{k^{j}}
$$

This ansatz is easily proved by induction, and the $a_{j}^{(l)}$ coefficients are recursively calculated,

$$
\begin{gathered}
a_{j}^{(l+1)}=\frac{k^{l} a_{j}^{(l)}}{\left(k^{l+1}-k^{l}\right)}, \quad j=0,1,2, \ldots, l \\
a_{l+1}^{(l+1)}=\frac{\left(-\sum_{j=0}^{l} a_{j}^{(l+1)} N^{k^{j}}\right)}{N^{k^{l+1}}} .
\end{gathered}
$$

These exact functions $N_{j}=N_{j}\left(N_{0}\right), j \neq 0$ can be used as the base of an elegant numerical method suited to compute the time-dependent solution of Eq. (3). This will be commented on in a forthcoming publication. In this paper, however, we will use Eq. (7) merely to test the accuracy of another approximated method, a discrete probabilistic one $112,18,19$, that will be used to solve Eq. (3). In this case of an ELS model with continuous damage, the elementary time step for one fiber to yield is given by [12]:

$$
\delta=\frac{1}{N_{0} f^{\rho}+N_{1}(a f)^{\rho}+N_{2}\left(a^{2} f\right)^{\rho}+\ldots+N_{n}\left(a^{n} f\right)^{\rho}},
$$


with

$$
f=\frac{N}{N_{0}+a N_{1}+a^{2} N_{2}+\ldots+a^{n} N_{n}} .
$$

Thus, $\delta$ is the inverse of the total "decay width" of the system. The total decay width is the sum of the contribution of all the lists $j=0,1, \ldots, n$. And each list contributes with a term

$$
\Gamma_{j}=N_{j} \sigma_{j}^{\rho}=N_{j}\left(a^{j} f\right)^{\rho} .
$$

The probability that the individual failure takes place in the list $j$ is equal to $p_{j}=\Gamma_{j} \cdot \delta$. With this natural assignment of probabilities, it is apparent that $\sum_{j=0}^{n} p_{j}=$ 1. In the process of breaking, a set of $N$ elements and $n$ allowed partial yields, we will have a total of $N(n+1)$ deltas, whose sum $\sum_{i=1}^{N(n+1)} \delta_{i}=T$, is the lifetime of the bundle. This method starts from an initial state, $\left(N_{0}, N_{1}, N_{2}, \ldots, N_{n}, N^{\prime}\right)=(N, 0,0, \ldots, 0,0)$ and ends in $(0,0,0, \ldots, 0, N)$.

The results obtained with this method are shown in Figs. 13. In each simulation, one has to fix the vector $(N, \rho, a, n)$, under the hypothesis that $F=N$ and $Y=1$. The standard model is obtained by setting $n=0$. In Fig. 1, for a vector $\left(10^{4}, 2,0.8,4\right)$, we compare the prediction of this method for $N_{1}\left(N_{0}\right)$ and $N_{2}\left(N_{0}\right)$ with the analytic result expressed in equation (7). It is apparent that the agreement is excellent. In Fig. 2, the behavior of the bundle strain, $f$, as a function of time, is plotted for the same vector of parameters as before but considering also the cases of $n=0$ and $n=1$. Here, one appreciates how the hypothesis of continuous damage leads to increasing the bundle's lifetime. It is interesting to note that at the time at which the system collapses, the relation $F=a^{n} f_{c}$ holds, i.e., the ultimate strain of the bundle, $f_{c}$, is related to the external load imposed over the system through the factor $a^{-n}$. This is also the case for the static continuous damage model [14] but substituting $F$ by $F / N$. Finally, Fig. 3 shows the behavior of the bundle's strain near the point of macroscopic rupture. It turns out that the strain scales with the distance to the critical point, $T-t$, as $f \sim(T-t)^{\beta}$ with $\beta=-\frac{1}{\rho}$. This result is universal, i.e., the material approaches its macroscopic collapse in this way regardless of the number of allowed partial yields. Universal behaviors are quite important in fracture processes because of the intrinsic and unavoidable sample to sample variations. Thus, although the lifetime of the bundle varies from one realization to another, the strain of the bundle would satisfy, for a given $\rho$ value, the same scaling function near the point of macroscopic collapse regardless of $n$. It is worth noting that this feature is not observed only in our simple model. For example, in a similar (in spirit) although more complicated model where visco-elastic cells are introduced, the same behavior near the critical point is found [20].

Up to now, we have assumed that the external load acting on the bundle is $F=N$. If we consider that $F$ is modified in a factor $\phi, F_{\phi}=\phi N$, then the lifetime of reference $T$ would be modified in the following way,

$$
T_{\phi}=\frac{T}{\phi^{\rho}} .
$$

Thus, we see that this exact property that works for the standard dynamic FBM with any load transfer rule and the power-law breaking rate [9] extends to the continuous damage version of this model.

Throughout this paper, we have considered brittle failure after $n$ damage events. Nevertheless, in order to describe macroscopic strain hardening instead of global failure, we should allow the fibers to have a $a^{n}$ residual stiffness after having yielded $n$ times.

Figure 4 shows the creep compliance $J(t)$, defined as $J(t)=f(t) / F$, as a function of dimensionless time $t$ for different values of $n$. It can be seen that the model qualitatively reproduces the behavior of $J(t)$ observed in experiments on amorphous materials [21]. For short times, the compliance is very slowly time dependent. As time passes, $J(t)$ becomes very strongly time dependent and finally the system approaches a plastic state for which the compliance is again very slowly time dependent. This confirms that the behavior of $J(t)$ depends on the time scale of the experiment 21].

In short, we have introduced a novel time dependent model of fracture with continuous damage for the breakdown of materials. The model was formulated in terms of a set of coupled non-linear differential equations and the time evolution of the system was studied by applying a discrete probabilistic method. This model is potentially useful to describe some elasto-plastic behaviors observed in real material fracture processes. Besides, it could guide our understanding to more complex time dependent models of fracture with continuous damage.

A.F.P thanks Javier Ribera for discussions. Y.M thanks A. Vespignani, H. J. Herrmann and F. Kun for stimulating discussions on our model, and the Abdus Salam International Centre for Theoretical Physics for hospitality and financial support. This work was supported in part by the Spanish DGICYT Project PB981594.

[1] Statistical Models for the Fracture of Disordered Media. Editors, H.J. Herrman and S. Roux, North Holland (1990), and references therein.

[2] Statistical Physics of Fracture and Breakdown in Disordered Systems. B. K. Chakrabarti, L. G. Benguigui, Clarendon Press, Oxford (1997), and references therein.

[3] A. Garcimartin, A. Guarino, L. Bellon, S. Ciliberto, Phys. Rev. Lett. 79, 3202 (1997).

[4] C. Maes, A. van Moffaert, H. Frederix, H. Strauven, Phys. Rev. B 57, 4987 (1998). 
[5] A. Petri, G. Paparo, A. Vespignani, A. Alippi, M. Constantini, Phys. Rev. Lett. 73, 3423 (1994).

[6] S. Zapperi, P. Ray, H. E. Stanley, A. Vespignani, Phys. Rev. Lett. 78, 1408 (1997).

[7] Y. Moreno, J. B. Gomez, A. F. Pacheco,Phys. Rev. Lett. 85, 2865 (2000).

[8] B.D. Coleman, J. Appl. Phys. 28, 1058 (1957); ibid 28, 1065 (1957).

[9] S.L. Phoenix and L. Tierney, Eng. Frature Mech. 18, 193 (1983).

[10] W.I. Newman, A.M. Gabrielov, T.A. Durand, S.L. Phoenix and D.L. Turcotte, Physica D77, 200 (1994).

[11] W.I. Newman, D.L. Turcotte and A.M. Gabrielov, Phys. Rev. E 52, 4827 (1995).

[12] M. Vázquez-Prada,J. B. Gómez, Y. Moreno, A. F. Pacheco, Phys. Rev. E 60, 2581 (1999).

[13] S. Zapperi, A. Vespignani, and H. E. Stanley, Nature 388, 658 (1997).

[14] F. Kun, S. Zapperi, H. J. Herrmann, Eur. Phys. J. B17, 269 (2000).

[15] A. E. Naaman, H. W. Reinhardt, High performance fiber reinforced cement composites (E and FN Spon, London, 1995).

[16] A. G. Evans, J. M. Domergue, E. Vagaggini, J. Am. Cerramic Soc. 77, 1425 (1994).

[17] T. Kanada, V. C. Li, J. Eng. Mech. 125, 290 (1999).

[18] W.A. Curtin and H. Scher, Phys. Rev. Lett. 67, 2457 (1991).

[19] J.B. Gómez, Y. Moreno, A. F. Pacheco, Phys. Rev. E. 58, 1528 (1998)

[20] F. Kun, private communication.

[21] D. Froelich, in Plastic deformation of amorphous and semi-crystalline materials, edited by B. Escaig and C. G'Sell, (Les Editions de Physique, Les Ulis, France) 1982.

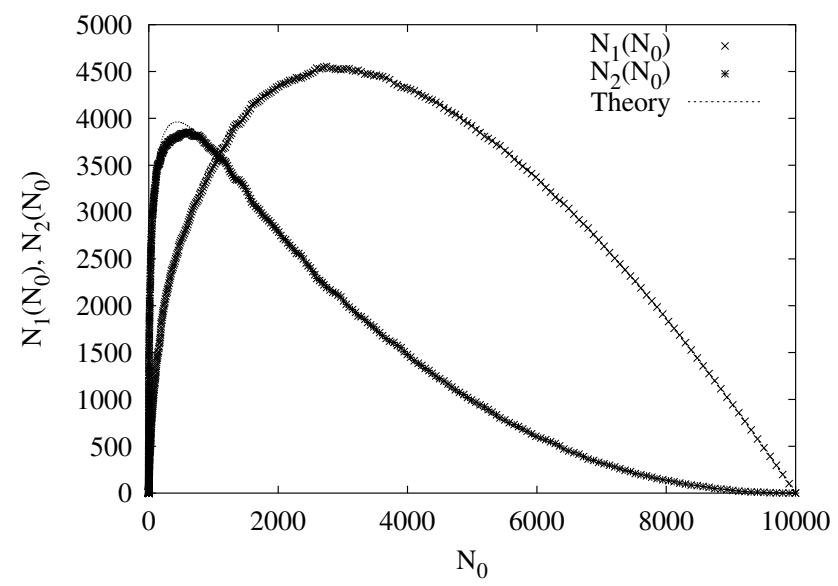

FIG. 1. Results of the probabilistic method (dotted curves) compared with the analytical prediction Eq. (7). The parameters used in the simulation are $N=10^{4}, a=0.8$, and $\rho=2$. The fibers are allowed to break $n=4$ times.

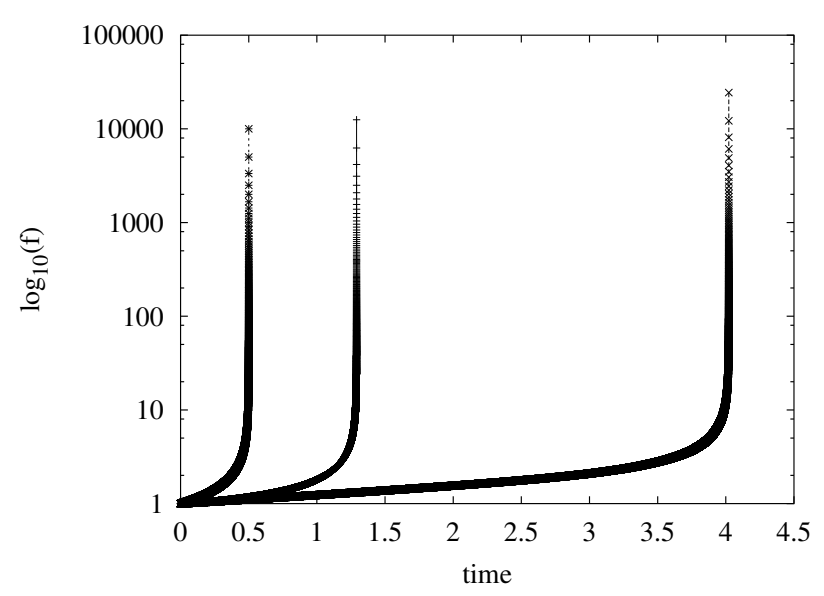

FIG. 2. Bundle's strain $(f)$ as a function of dimensionless time for different values of $n$ (from left to right $n=0,1,4$ ). The model parameters are the same as Fig 11. Multiple failures lead to increase in the lifetime of the bundle.

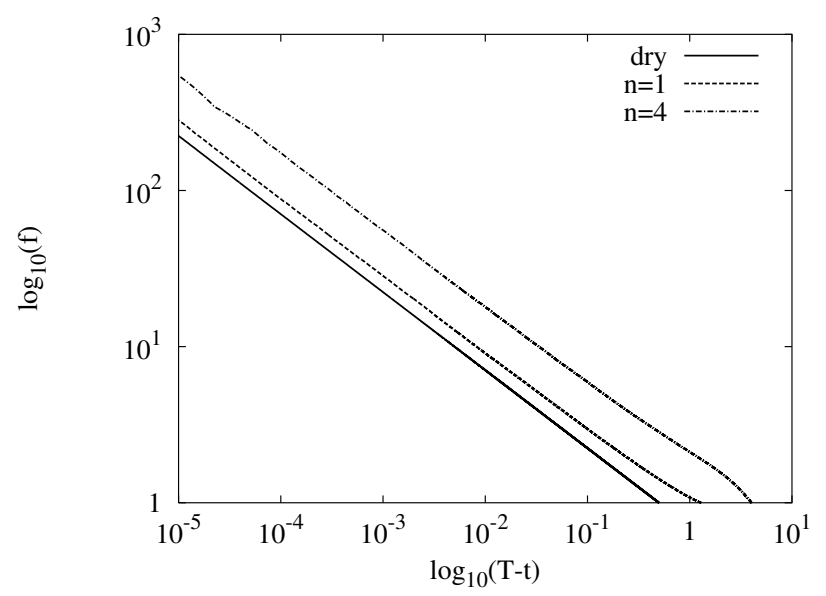

FIG. 3. Behavior of the material strain near the macroscopic point of rupture for $N=10^{4}, a=0.8, \rho=2$ and the values of $n$ indicated in the figure. The scaling relation satisfies $f \sim(T-t)^{\beta}$, with $\beta=-\frac{1}{\rho}$.

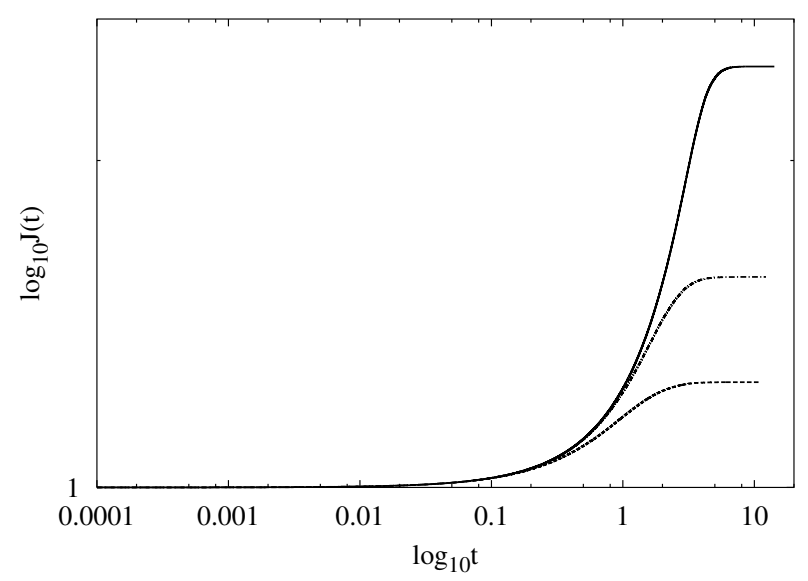


FIG. 4. The creep compliance $J(t)$ as a function of dimensionless time for different values of $n(n=1,2,4$ as $J$ increases). The behavior is qualitatively the same as that obtained in creep experiments on amorphous materials. 